\title{
SOME SIMILARITY SOLUTIONS FOR REACTION ACCOMPANYING MASS TRANSFER ACROSS RAPIDLY MOVING INTERFACE
}

\author{
TsuTOMu HIROSE \\ Department of Chemical Engineering, Kyushu University, \\ Fukuoka, 812
}

This article may be considered a supplement of the paper by Chakrabarti and Ghoshal ${ }^{4)}$ titled "Group Theoretic Approach to Similarity Solutions of Chemical Boundary Layer" which appeared in this Journal recently. The stretching invariance employed by them was restricted to reactions of other than first order. In the present article, the author shows that the problem with first-order reaction across a rapidly moving interface is reduced to a heat conduction equation to which the invariance by infinitesimal transformations developed by Bluman and $\mathrm{Cole}^{2)}$ can be applied directly. Two examples are given, one of which is a new solution and the other a different approach to an established solution.

\section{General Theory}

A convective diffusion equation assumes the following form in terms of appropriately normalized variables:

$$
P e\left(u \frac{\partial C}{\partial x}+v \frac{\partial C}{\partial y}\right)=\frac{\partial^{2} C}{\partial y^{2}}-D a C
$$

in which $D a$ is Damköhler number, $k L^{2} / D$. For mass transfer across a rapidly moving interface with high $P e$, the whole velocity profile may be approximated by interfacial velocity $u_{0}$ and one gets ${ }^{6)}$

$$
\begin{aligned}
& u=u_{0}=u_{0}(x) \\
& v=-\frac{1}{r^{j}} \frac{\partial}{\partial x}\left(r^{j} u_{0} y\right)
\end{aligned}
$$

where $r$ is the distance from the axis in axisymmetric flow, and $j=0$ for plane flow and $j=1$ for axisymmetric flow.

Defining new variables $z, t$ and $g$ as

$$
\begin{aligned}
& z=z(y, x)=r^{j} u_{0} y \\
& t=t(x)=(1 / P e) \int u_{0} r^{2 j} \mathrm{~d} x \\
& g=g(z, t)=C \exp \left(\int m \mathrm{~d} t\right)
\end{aligned}
$$

where

$$
m=m(t)=D a /\left(r^{j} u_{0}\right)^{2}
$$

then one gets formally a heat equation

Received November 4, 1975.

$$
\frac{\partial g}{\partial t}=\frac{\partial^{2} g}{\partial z^{2}}
$$

with varying boundary conditions

$$
\begin{aligned}
g(0, t) & =\exp (\oint m \mathrm{~d} t) \\
g(\infty, t) & =0
\end{aligned}
$$

and an appropriate initial condition at $t=0$.

Bluman and $\mathrm{Cole}^{2}$ found the general similarity solution of Eq. (8) based on the group invariance by infinitesimal transformations. They show that if

$$
\begin{aligned}
g^{*} & =g+\varepsilon \eta(z, t, g)+O\left(\varepsilon^{2}\right) \\
z^{*} & =z+\varepsilon \xi(z, t, g)+O\left(\varepsilon^{2}\right) \\
t^{*} & =t+\varepsilon \tau(z, t, g)+O\left(\varepsilon^{2}\right)
\end{aligned}
$$

is a one-parameter Lie group leaving invariant Eq. (8), functions $\eta, \xi$ and $\tau$ must take the form of

$$
\begin{aligned}
& \eta=f(z, t) g \\
& \xi=\xi(z, t)=\kappa+\delta t+\beta z+\gamma z t \\
& \tau=\tau(t)=\alpha+2 \beta t+\gamma t^{2} \\
& f=f(z, t)=-(\gamma / 4)\left(z^{2}+2 t\right)-\delta z / 2+\lambda
\end{aligned}
$$

and that a new independent (similarity) variable $\zeta$ and a dependent variable $F(\zeta)$, respectively, are given as integral constants of the first and the second equality of the following characteristic equation

$$
\frac{\mathrm{d} z}{\xi}=\frac{\mathrm{d} t}{\tau}=\frac{\mathrm{d} g}{f g}
$$

\section{Stagnation Flow at Spherical Bubble or Drop}

A subgroup of Eqs. (11) (13) must leave invariant the boundary and initial conditions to apply the above similarity to individual problems. Invariance of $z=0$ and $t=0$ implies that

$$
\begin{array}{lll}
z^{*}=z+\varepsilon \xi(z, t)=0 & \text { at } & z=0 \\
t^{*}=t+\varepsilon \tau(t)=0 & \text { at } & t=0 \\
\text { i. e. } \quad \alpha=\kappa=\delta=0 & &
\end{array}
$$

To leave invariant the boundary condition Eq. (9),

$$
\begin{aligned}
& \begin{aligned}
g^{*} & =g+\varepsilon g f=\exp \left\{\int m^{*}\left(t^{*}\right) \mathrm{d} t^{*}\right\} \\
& =\exp \left(\int m \mathrm{~d} t\right)+\varepsilon \tau m \exp \left(\int m \mathrm{~d} t\right) \text { at } z=0
\end{aligned} \\
& \text { i. e. } \quad m=\frac{f}{\tau}=\frac{\gamma t / 2+\lambda}{2 \beta t+\gamma t^{2}}
\end{aligned}
$$


Thus, the similarity solution exists only if the function $m$ takes the form of Eq. (24). One such applicable case is the flow near axisymmetric stagnation point in creeping or inviscid flow around a spherical bubble or drop of which the interface is rapidly circulating. In this case ${ }^{6)}$

$$
u_{0}=b x \quad \text { and } \quad r=x \quad(b: \text { Constant })
$$

and Eqs. (5) and (7) give

$$
m=p / t
$$

where

$$
p \equiv \frac{\lambda}{2 \beta}=\frac{D a / P e}{4 b}
$$

This problem is reduced to a stretching invariance in the transformations of

$$
\begin{aligned}
& \xi=\beta z \\
& \tau=2 \beta t \\
& f=2 \beta p
\end{aligned}
$$

which in turn gives new variables by solving Eq. (18)

$$
\begin{gathered}
\zeta=z / \sqrt{2 t} \\
g=t^{p} F(\zeta) \\
\frac{\mathrm{d}^{2} F}{\mathrm{~d} \zeta^{2}}+\zeta \frac{\mathrm{d} F}{\mathrm{~d} \zeta}-2 p F=0
\end{gathered}
$$

The solution satisfying boundary conditions is

$$
\begin{aligned}
C & =F t^{p} \exp \left(-\int m \mathrm{~d} t\right)=F \\
& =\frac{U(1 / 2+2 p, \zeta)}{U(1 / 2+2 p, 0)} \exp \left(-\frac{\zeta^{2}}{4}\right)
\end{aligned}
$$

in which $U$ is Weber parabolic cylinder function ${ }^{1}$. When $2 p$ is an integer, Eq. (34) becomes Carslaw's solution to a heat conduction equivalent. Enhancement factor $E$ is calculated by

$$
\begin{aligned}
E & =\left(\frac{\partial C}{\partial y}\right)_{p=p} /\left(\frac{\partial C}{\partial y}\right)_{p=0} \text { at } y=0 \\
& =\frac{\Gamma(1 / 2) \Gamma(1+p)}{\Gamma(1 / 2+p)}
\end{aligned}
$$

Since physical mass transfer rate at stagnation point is described by

$$
S h=4 \sqrt{P e / \pi b}
$$

the parameter $p$ is related to the familiar modulus $M$ by

$$
p=M / \pi \quad \text { where } \quad M=k D / k_{L}^{2}
$$

of which the substitution into Eq. (36) produces an $E$ vs. $\sqrt{M}$ correlation chart.

\section{Uniform Interfacial Velocity or Penetration Model}

In this case $m$ is constant and Eq. (24) does not allow a similarity solution. So a superposition of similarity solutions is sought. Putting $\alpha=\gamma=\kappa=$ $\lambda=0$, one gets the following subgroup of Eqs. (15) (17):

$$
\xi=2 p t+z
$$

$$
\begin{aligned}
& \tau=2 t \\
& f=-p z \text { where } p=\delta / 2 \beta
\end{aligned}
$$

The parameter $p$ is an arbitrary constant and one seeks first the eigenfunctions $F_{i}(\zeta)$ corresponding to the eignevalues $p_{i}$. New variables $F_{i}$ and $\zeta$ are derived from Eq. (18) with Eqs. (39) (41) as follows:

$$
\begin{gathered}
\zeta=z / \sqrt{t}-2 p_{i} \sqrt{t} \\
g_{i}=F_{i}(\zeta) \exp \left\{-\left(p_{i}^{2} t+p_{i} \zeta \sqrt{t}\right)\right\} \\
\frac{\mathrm{d}^{2} F_{i}}{\mathrm{~d} \zeta^{2}}+\frac{1}{2} \zeta \frac{\mathrm{d} F_{i}}{\mathrm{~d} \zeta}=0
\end{gathered}
$$

The superposition of these solutions yields

$$
g=\sum_{i=0}^{\infty} A_{i} \operatorname{erfc}(\zeta / 2) \exp \left\{-\left(p_{i}^{2} t+p_{i} \zeta \sqrt{t}\right)\right\}
$$

At the interface i.e., at $\zeta=-2 p_{i} \sqrt{t}$, this becomes

$$
\exp (m t)=\sum_{i=0}^{\infty} A_{i} \operatorname{erfc}\left(-p_{i} \sqrt{t}\right) \exp \left(p_{i}^{2} t\right)
$$

from which $p_{i}$ 's and $A_{i}$ 's are found to be

$$
p_{0}=\sqrt{m}, p_{1}=-\sqrt{m}, A_{0}=A_{1}=1 / 2, A_{i}=0 \text { for } i \geqq 2
$$

The concentration distribution is obtained finally in the form of

$$
\begin{aligned}
C= & \frac{1}{2} \exp (-\sqrt{m} z) \operatorname{erfc}\left\{\frac{z}{2 \sqrt{t}}-\sqrt{m t}\right\} \\
& +\frac{1}{2} \exp (\sqrt{m} z) \operatorname{erfc}\left\{\frac{z}{2 \sqrt{t}}+\sqrt{m t}\right\}
\end{aligned}
$$

This equation is identical with Danckwerts ${ }^{5}$-Carslaw's ${ }^{3)}$ solution by the use of Duhamel's theorem.

\section{Concluding Remarks}

Permissible boundary conditions are seriously restricted due to the addition of a reaction term to the diffusion equation as shown by Eq. (24). Although similarity solutions are not limited to the two examples given here, one cannot expect so many solutions as are obtained under non-reacting conditions.

\section{Literature Cited}

1) Abramowitz, M. and I. A. Stegun, (ed.): "Handbook of Mathematical Functions with Formulas, Graphs and Mathematical Tables", p. 686, U.S. National Bureau of Standards (1964).

2) Bluman, G. W. and J. D. Cole: J. Math. Mech., 18, 1025 (1969); Quart. Appl. Math., 31, 403 (1974); and "Similarity Methods for Differential Equations", SpringerVerlag (1974).

3) Carslaw, H. S. and J. C. Jaeger: "Conduction of Heat in Solids”, p. 63, 2nd ed., Oxford University Press (1959).

4) Chakrabarti, K. M. and S. K. Ghoshal: J. Chem. Eng. Japan, 8, 74 (1975).

5) Danckwerts, P. V.: Trans. Farad. Soc., 46, 300 (1950).

6) Lochiel, A. C. and P. H. Calderbank: Chem. Eng. Sci., 19, 471 (1964). 